\title{
Quaderni
}

QUADERN I Communication, technologies, pouvoir

\section{« Haro sur l'affichage! » Agir par écrit contre la publicité dans l'espace public parisien}

\section{Clara Lamireau}

\section{(2) OpenEdition \\ 1 Journals}

\section{Édition électronique}

URL : http://journals.openedition.org/quaderni/477

DOI : 10.4000/quaderni.477

ISSN : 2105-2956

\section{Éditeur}

Les éditions de la Maison des sciences de l'Homme

\section{Édition imprimée}

Date de publication : 5 avril 2010

Pagination : 19-30

\section{Référence électronique}

Clara Lamireau, « «Haro sur l'affichage! » Agir par écrit contre la publicité dans l'espace public parisien », Quaderni [En ligne], 72 | Printemps 2010, mis en ligne le 05 avril 2012, consulté le 21 décembre 2020. URL : http://journals.openedition.org/quaderni/477 ; DOI : https://doi.org/10.4000/ quaderni. 477 


\section{$D$ ossier}

\section{" haro sur \\ l'affichage !" \\ Agir \\ par écrit \\ contre la \\ publicité \\ dans l'espace \\ public parisien}

Les mouvements contestataires axés sur la dénonciation de la publicité comme système de propagande sont l'objet, depuis le début des années 2000, d'une attention médiatique et éditoriale. Bien que les actions de contestation de la publicité soient attestées dans plusieurs pays du monde dès les années $1970^{1}$, leur succès médiatique est conjugué, en France, à deux événements : la traduction de l'ouvrage de Naomi Klein, No Logo : La tyrannie des marques, aux éditions Actes Sud en $2001^{2}$ et la vague d'actions spectaculaires à l'encontre de l'affichage publicitaire dans le métro parisien, en automne 2003.

Ces mouvements anti-publicitaires sont décrits par les auteurs - pour la plupart journalistes attentifs à l'évolution des mouvances contestataires sur le sol français - comme participant à l'émergence de «nouvelles » formes de protestation. Sous le titre explicite Les nouveaux militants, Laurent Jeanneau et Sébastien Lernould affirment ainsi que les actions anti-publicitaires sont menées par des personnes ayant fui les luttes politiques et syndicales traditionnelles pour s'engager sur

Clara Lamireau

Post-doctorante Équipe "Anthropologie de l'écriture" IIAC - EHESS des chemins plus «festifs", provocateurs et trublions ${ }^{3}$. De la même façon Sébastien Darsy, avec Le Temps de l'Anti Pub, inscrit les actions anti-publicitaires dans une tendance de "politisation des modes de vie» et une aspiration à de nouvelles valeurs, à une prise de conscience des méfaits de l'extension d'une communication commerciale dans l'espace public ${ }^{4}$.

Or ces analyses, bien que très détaillées dans la différenciation des mouvances d'un même phénomène (en l'occurrence, la contestation anti-publicitaire), semblent totalement négliger à la fois l'histoire sociale dont ces mouvements 
sont issus et l'espace actuel concret dans lequel ils agissent. Mon propos ne sera pas ici de revenir sur cette histoire de la contestation ${ }^{5}$, mais je souhaite prendre en considération l'hypothèse développée par Charles Tilly selon laquelle « toute population a un répertoire limité d'actions collectives, c'està-dire de moyens d'agir en commun sur la base d'intérêts partagés $»^{6}$. La notion de « répertoire d'actions collectives » est pour cet historien et sociologue une simple métaphore, qui connaîtra pourtant un succès académique retentissant. Dans son ouvrage fondateur La France conteste : de 1600 à nos jours, il avance ainsi prudemment l'usage d'une telle notion: "Il se peut que le mot "répertoire" ne soit qu'une étiquette sous laquelle ranger tous ceux à quoi les gens recourent pour atteindre des buts communs. (...) Ces différents moyens d'actions composent un répertoire, un peu au sens où on l'entend dans le théâtre et la musique $»^{7}$. Les actions anti-publicitaires actuelles ne seraient donc pas tout à fait « nouvelles » : malgré la spontanéité qui leur est attribuée, les militants anti-publicitaires agissent dans un cadre déterminé, qu'ils actualisent euxmêmes de façon consciente, sachant qu'ils plongent et innovent dans un « répertoire » déjà établi. Davantage que des « inventions », nous sommes donc plutôt face à des « interprétations », au sens musical du terme, sur la base de partitions et de principes politiques existants. Mais plutôt que de routiniser l'usage du terme de « répertoire », il est sans doute préférable de parler de «pratiques » protestataires, quitte à garder à l'esprit les questions qu'engage l'idée de répertoire d'action.

La seconde négligence des analyses médiatiques du mouvement anti-publicitaire est liée à une conception trop élargie de la notion d'espace public.
Héritières d'une philosophie politique de l'agora pensée comme un espace de la cohésion sociale ${ }^{8}$, ces analyses tendent à confondre la sphère publique et l'espace public comme espace physique de la ville. Or la sociologie urbaine a su au contraire démontrer que le propre de l'espace public est son caractère " disputable » ${ }^{9}$. L'hétérogénéité des points de vue, la porosité du domaine privé et du domaine public, l'acceptation différenciée des informations circulant dans l'espace urbain en font un lieu de débats, de concurrences et de conflits. Plutôt que de parler d'espace public au sens abstrait du terme, il semble donc plus pertinent de s'interroger sur la diversité des pratiques concrètes qui jalonnent cet espace. Ce sont en effet ces pratiques, ces usages, les interactions qui s'y déroulent et les règles qui l'encadrent, qui font qu'un espace est public et qu'un autre ne l'est pas.

À travers l'étude d'une action spécifique mise en œuvre par un groupe anti-publicitaire, il s'agit de comprendre comment les luttes de pouvoir s'inscrivent concrètement dans l'espace physique de la ville : toute action engagée dans l'espace public - ici, par le biais de l'écriture - contient une réflexion sur le bien public, le bien commun et la co-production de civilités. Partant de cette hypothèse, je m'intéresserai à la façon dont les militants anti-publicitaires agissent par l'écriture dans l'espace public. L'approche ethnographique choisie, et en particulier l'analyse des situations de production, de circulation et de médiatisation des écrits contestataires, dévoile tout autre chose que les aspirations politiques d'une époque ou l'idéologie d'un groupe d'activistes. À travers l'observation des préparations d'une action de graffitage anti-publicitaire et celle des activités 
concrètes des militants engagés dans cette action, l'analyse s'attache à identifier comment cette force accordée à l'affichage publicitaire est à l'origine d'une contre-action d'écriture exposée, dans un jeu de conventions, de détournements, de tactiques tenant compte de savoirs sur les médias et la communication politique ${ }^{10}$. Le questionnement porte ici une attention particulière à l'action réalisée, à sa préparation, son accomplissement, ses prolongements et son encadrement, sa médiatisation. En quoi l'écriture permet-elle l'accomplissement d'une action contestataire? Quelles sont les conditions requises pour que ces écrits exposés ou diffusés dans l'espace public (graffitis, pancartes et tracts) soient performatifs ?

Ce questionnement amène à considérer l'écriture dans une perspective pragmatique et à examiner l'hypothèse selon laquelle il existerait des « actes d'écriture ». Cette notion, forgée sur le modèle de l'expression « acte de langage » de John Austin ${ }^{11}$, qui démontre comment des énoncés peuvent être en eux-mêmes l'acte qu'ils désignent. Ces énoncés, qu'il nomme « performatifs », ne décrivent pas un fait mais « font» quelque chose, dans un cadre qu'Austin cantonne, dans ses premiers exemples, à un contexte rituel et institutionnel, comme le mariage («Oui»-je prends cette femme comme épouse légitime -) ou le baptême («Je te baptise »). Proférer ces phrases, c'est accomplir un acte. La notion d'acte d'écriture explore l'idée de la performativité dans un contexte où l'énonciation n'est pas orale mais écrite, avec toutes les spécificités qu'elle peut alors comporter. Un acte d'écriture, serait donc, selon la proposition de Béatrice Fraenkel ${ }^{12}$ :

- un acte graphique (ou le geste de la personne qui écrit) ;

- la fabrication d'un objet écrit (une affiche publicitaire graffitée) ;

- un acte illocutoire, c'est-à-dire une action accomplie par cette énonciation écrite (dans le cas du « barbouillage » que nous allons étudier, il s'agit à la fois de salir et de rendre publique cette souillure).

Écrire, c'est produire un énoncé mais aussi réaliser un ensemble de gestes, fabriquer un objet et poser un acte.

\section{La confrontation à la loi par la maîtrise d'un dispositif graphique}

Le mode d'action privilégié par ces militants est ce qu'ils désignent sous le terme de «barbouillage ». Le terme n'est pas choisi au hasard : « barbouiller », c'est tacher, maculer, salir un support avec un produit couvrant, comme la peinture. Pour les militants qui emploient ce terme, il s'agit de salir un support publicitaire, de s'attaquer physiquement à un type d'écriture exposée ${ }^{13}$ jugée insupportable. Le « barbouilleur», avant d'être un scripteur contestataire, est donc un lecteur de la ville, confronté à un type d'inscription qui ne devrait pas, selon lui, exister. Pourtant, les « barbouilleurs » ne se contentent pas de faire des taches de peinture sur l'affiche publicitaire ou de la recouvrir : ils y ajoutent des inscriptions. Nous allons tenter de comprendre comment se construit, au sein de ce groupe militant, une intervention graphique illégale dans l'espace public, et comment cette intervention peut être un moyen de se confronter à la loi ${ }^{14}$, de provoquer un procès afin de porter le débat de la rue jusqu'au tribunal correctionnel. 
Revenons dans un premier temps sur le déroulement d'un «barbouillage ». Chaque mois, un rassemblement est organisé à la sortie d'une station de métro. Le lieu de rendez-vous, d'abord tenu secret, est ensuite diffusé sur un site Internet et communiqué à la presse. Les militants et sympathisants se retrouvent à l'horaire donné, les journalistes sont déjà présents avec leur matériel. Un ou deux militants prennent la parole pour énoncer leurs revendications et la philosophie non-violente de leurs actions, entourés d'autres personnes qui face à la presse brandissent des pancartes sur lesquelles sont inscrites des slogans : «Publicité = violence », « Non à la pollution visuelle ». Puis le groupe, composé le 50 à 80 personnes, converge vers les panneaux publicitaires préalablement choisis. Trois à six personnes s'emparent alors de bombes de peinture et inscrivent des slogans sur les publicités. Pendant ce temps, la police, prévenue à l'avance par l'annonce sur le site Internet, s'approche des graffiteurs et procède aux interpellations. Les graffiteurs ont déjà leur pièce d'identité en main et se laissent interpeller, sous les applaudissements des sympathisants. Des chants sont entonnés, puis la dispersion générale est organisée par les militants eux-mêmes.

Deux aspects de cette action contestataire peuvent être ici soulignés. Le rapport aux forces de l'ordre, étonnament serein, est issu d'une réflexion sur la non-violence, elle-même utilisée comme ressource au sein d'un répertoire d'action. Comment ce choix de la non-violence transparaît dans l'usage de l'écrit au sein de ces actions ? Les « barbouillages », dont le but officiel est de modifier la loi sur l'affichage publicitaire, sont construits comme des événements publics.
Comment la circulation de l'écriture exposée contestataire est-elle alors maîtrisée à destination des médias?

Le premier étonnement de l'observateur face à ce type d'action anti-publicitaire est le rapport entretenu par les graffiteurs, qui commettent une action illégale, et les forces de police. Les « barbouilleurs » agissent au grand jour et à visage découvert, sans chercher à se cacher ou à fuir. Pourtant, l'histoire récente du mouvement antipublicitaire à Paris est marquée de confrontations plus violentes. En 2003, des actions anti-publicitaires dans le métro parisien sont organisées de façon massive. Des centaines de personnes descendent dans plusieurs stations, pinceaux et bombes de peinture en main, pour recouvrir en quelques minutes les affiches de graffitis, en scandant des slogans contre la police. Les interpellations sont nombreuses et en 2004, un procès est organisé contre 62 graffiteurs présumés. Depuis 2005 , les groupes informels de graffiteurs antipublicitaires s'organisent et se judiciarisent. Le «Collectif des déboulonneurs » ${ }^{15}$ est créé. Les procès, au lieu d'être craints, sont désormais préparés comme des débats publics. L'acte d'écriture qui consiste à inscrire des slogans sur des affiches publicitaires est ainsi qualifié par les militants comme acte de « désobéissance civile », concept juridique élaboré par le philosophe Henry-David Thoreau $^{16}$. "La désobéissance civile peut être définie comme un acte public, non violent, décidé en conscience, mais politique, contraire à la loi et accompli le plus souvent pour amener un changement dans la loi ou bien la politique $d u$ gouvernement $»{ }^{17}$. Cette qualification de $«$ désobéissance civile » implique certaines précautions de la part des militants : pour que l'action soit 
efficace, il faut lui donner un cadre. Le cadre choisi et revendiqué par les militants est ici celui de la « non-violence », notamment vis-à-vis des forces de l'ordre.

La prise en compte de cet environnement de l'action est fondamentale pour comprendre l'ensemble des actes qui la composent ou, selon l'expression d'Erving Goffman, pour comprendre « ce qui est en train de se passer ». En effet, si nous voulons sortir d'une appréhension de l'action contestataire uniquement centrée sur la question de l'idéologie, nous devons regarder de plus près l'activité en train de se faire, les situations de production et de réception de ces actions. Ainsi, une action contestataire implique, du point de vue de ses acteurs, une démarche dans laquelle ils sont engagés, et diffère d'une autre situation de la vie quotidienne. Goffman propose la notion de « cadre » (frame) pour définir « les principes d'organisation qui structurent les événements et notre propre engagement subjectif $\gg^{18}$. Dans le cadre d'une action de protestation, les militants seront d'abord engagés dans un « cadre primaire » : « Est primaire un cadre qui nous permet, dans une situation donnée, d'accorder du sens à tel ou tel de ses aspects, lequel autrement serait dépourvu de signification $»^{19}$. Pour Goffman, les cadres primaires sont essentiellement les cadres naturels, purement physiques et les cadres sociaux, qui sont le fait d'actions ou d'intentions humaines. Mais ce cadre primaire ne suffit pas, ni du point de vue de l'observateur, ni du point de vue de l'acteur, pour comprendre de ce qui se dit, ce qui se fait et ce qu'il se passe. Déterminer la catégorie d'action (ou d'interaction) dans laquelle on se situe, passera davantage par les « modes » et « modalisations ». Un mode est « un ensemble de conventions par lequel une activité donnée, déjà pourvue d'un sens par l'application d'un cadre primaire, se transforme en une autre activité qui prend la première pour modèle mais que les participants considèrent comme sensiblement différente $»^{20}$. Dans les exemples de « modes fondamentaux » que propose Goffman se trouvent les cérémonies, qui déplacent les interactions sur un terrain rituel : " il s'y passe quelque chose qui diffère des activités ordinaires sans qu'on sache précisément quoi $\gg^{21}$.

Nous formulons ici l'hypothèse que les «barbouillages » organisés par le «Collectif des Déboulonneurs » se préparent et s'accomplissent selon un mode cérémoniel, sur la base d'un cadre général tenant compte de l'espace physique de la ville - l'espace public de la rue - et d'une philosophie non violente. Au sein de ce mode cérémoniel, l'écrit est à la fois une ressource et un moyen de rendre visible cette action par le plus grand nombre - en s'appuyant sur des relais médiatiques.

Ce cadre permet de rassembler autour d'une même action des individus issus d'expériences et d'horizons politiques variés, de militants écologistes aguerris aux étudiants pour qui cet engagement constitue une première initiation. Mais davantage que les «barbouilleurs » euxmêmes - terme désignant les personnes écrivant sur les affiches - l'énonciation des principes de non-violence permet d'encadrer les personnes assistant à l'action sans y prendre part directement. Ces «soutiens » opèrent la fonction de public actif (applaudissant, chantant, pouvant s'engager dans des prises de parole à l'aide du mégaphone) et peuvent être composés aussi 
bien de militants inscrits dans la philosophie des «barbouillages » que de simples passants arrivés là par hasard - introduisant un élément d'incertitude quant aux réactions prévues. Une personne est donc préalablement désignée pour gérer les éventuelles perturbations dans le bon déroulement de l'action, prenant à part et tentant de raisonner - ou d'exclure - les individus qui ne répondraient pas à ce cadre.

Lors d'un « barbouillage », un tract est distribué à l'assistance, au début du rassemblement. Pour respecter la propreté des lieux et se conformer au Code de l'Environnement, un militant est chargé de ramasser ceux qui seraient jetés sur la voie publique. En effet l'article L541-10-1 stipule que " toute personne physique ou morale qui, gratuitement, (...) distribue des imprimés (...) dans les lieux publics ou sur la voie publique, est tenue de contribuer à la collecte, la valorisation et l'élimination des déchets ainsi produits. ». Puisque l'action des « barbouilleurs » vise à modifier le Code de l'Environnement pour limiter l'affichage publicitaire, les militants tiennent à ce que chaque acte du « barbouillage », exceptés les graffitis sur les affiches elles-mêmes, s'accorde à la loi.

Le tract ainsi distribué recommande une attitude particulière face aux forces de l'ordre : «On s'oppose à la publicité, pas à la police. (...) Ne pas s'interposer en cas d'intervention de la police, ne pas entonner de slogans contre la police, rester détendu et courtois $»^{22}$.

Il s'agit là encore de se conformer à la loi, pour que le seul acte illégal du « barbouillage » reste l'inscription de slogans sur les affiches publicitaires. Le Code Pénal prévoit en effet 7500 euros d'amende pour «provocation directe à la rébellion, manifestée soit par des cris ou des discours publics, soit par des écrits affichés ou distribués, soit par tout autre moyen de transmission de l'écrit, de la parole ou de l'image» (article 433-10).

Pour cette même raison, aucune résistance n'est opposée aux forces de l'ordre au moment de l'interpellation : les graffiteurs tendent volontairement leur carte d'identité ou leur passeport, en revendiquant leur acte.

Au moment de la réalisation des graffitis, c'est encore une fine connaissance de la loi qui guide l'action des militants. Les graffitis sont peints uniquement sur les affiches ou les vitres qui les abritent, propriété privée de l'afficheur. Aucune trace de peinture n'est décelable sur les surfaces environnantes et le mobilier urbain, afin que l'accusation porte uniquement sur l'affichage publicitaire $^{23}$.

De la même façon, le type d'inscription choisi doit être précisément identifié afin de ne pas laisser s'immiscer de confusion sur l'acte réalisé. La fiche de préparation d'une action de «barbouillage » précise ainsi : «Ne pas faire un tag mais écrire une inscription de son choix avec une bombe de peinture. Les tags relèvent d'une loi plus sévère que celle d'une dégradation par l'écriture d'une inscription. Vous êtes des barbouilleurs. Refusez l'appellation de tagueurs devant les journalistes et les policiers ». Le tag est donc un type d'inscription repoussoir auquel les militants anti-publicitaires, bien qu'utilisant les mêmes instruments d'écriture (la bombe), refusent d'être identifiés. D'une part parce que 
la loi dite Perben 2, portant adaptation de la justice aux « évolutions de la criminalité », prévoit des dispositifs spécifiques sur les dégradations commises en «bandes organisées $»^{24}$. D'autre part parce que les «barbouilleurs » doivent, pour être identifiés à part entière dans le discours médiatique, revendiquer la spécificité de leurs actes. Cette connaissance du cadre juridique de l'action et la formation permanente à laquelle s'astreignent les «barbouilleurs » est ce qui constitue la principale différence avec les actions massives des années précédentes - notamment de l'année 2003. Désormais, chaque acte est pensé en fonction de l'éventuel procès à venir.

Dans trois moments importants du « barbouillage », c'est donc la maitrise d'un dispositif graphique qui permet de donner un cadre non-violent à l'action. Avant la réalisation de l'acte graphique illégal (les graffitis), c'est un dispositif graphique légal (le tract donné de la main à la main et ramassé) qui avertit les participants. Pendant l'action, la maîtrise du geste graphique permet de limiter l'inscription de slogans à la cible visée. À la fin de l'action, les papiers d'identité des graffiteurs sont brandis face à la Police Nationale pour faciliter les arrestations. Si ces trois actes correspondent aux principes politiques des militants (écologie et non-violence), ils sont également un moyen d'encadrer le «barbouillage » et de préparer le procès des graffiteurs.

\section{Le «barbouillage », événement d'écriture public}

Les « barbouillages » sont construits comme des événements publics. Le relais médiatique de ces actions est donc méticuleusement préparé. Le
« Collectif des déboulonneurs » propose ainsi à ses militants une fiche de conseils pour entrer en contact avec les journalistes, ménager un effet d'attente sur le lieu de l'action et rédiger des communiqués de presse. Les « barbouilleurs » se constituent ainsi un carnet d'adresses de contacts spécialisés, invités à chaque manifestation. Une semaine à l'avance, un communiqué est envoyé, selon certaines règles informatives et typographiques : "il doit indiquer le type d'action, ses mobiles, le jour, l'heure, la ville. (...) Le titre ou thème général du communiqué doit être composé en très gros caractères gras ». Ce communiqué écrit est doublé d'une première vague de coups de téléphone. Trois jours avant l'action, le lieu précis est délivré sur un site Internet. Une heure avant l'action, une seconde vague de coups de téléphone est donnée.

La fiche de préparation de l'action du «barbouillage » délivre quelques orientations détaillées sur le meilleur moment pour organiser ce type d'action, en fonction de l'heure des journaux télévisés : «Le jour et l'heure sont choisis collectivement. On peut penser que le vendredi entre 12 heures et 14 heures, ou à 17 heures, est particulièrement bon pour le journal télévisé du soir, et les journaux sont encore en fabrication pour le samedi. Le samedi permet de croiser plus de passants, mais les journalistes travaillent plus rarement samedi ou dimanche... L'action n'a tout son sens que si la presse est présente ».

La presse n'est pas convoquée pour faire l'annonce publique de l'action, mais pour la relayer une fois réalisée. Les médias jouent un rôle fondamental dans l'action anti-publicitaire car ils permettent une diffusion de cette action au- 
delà des quelque 50 à 80 personnes présentes sur place. Ils reproduisent, à la télévision ou dans les journaux, les graffitis des barbouilleurs. L'effet visuel du «barbouillage" est pensé à destination des médias. Depuis 2006, un nouveau type d'écriture exposée est ainsi apparu: la pancarte. Cet affichage mobile en carton est réalisé sur place, au moment où les participants commencent à se rassembler autour de la station de métro. Il permet de soutenir visuellement les revendications précises des militants, lorsque ceux-ci prennent la parole avant de se rendre devant les affiches : tandis que l'un d'entre eux grimpe sur un tabouret, déclamant au mégaphone les raisons de son action, d'autres se tiennent à côté de lui, brandissant les pancartes. Dans ce dispositif, le support compte autant que le slogan dans la diffusion du message : les pancartes ont une taille précise, 50 centimètres sur 70 centimètres. C'est exactement la taille qui est concédée dans le Code de l'Environnement cité plus haut pour l'affichage associatif dans l'espace public. Les militants anti-publicitaires souhaiteraient que toutes les affiches soient réduites à ce format. Le slogan («50x 70 pour la liberté de réception») est donc redoublé par le support de l'inscription. Cette scène (le militant au mégaphone, les militants aux pancartes) est jouée face aux caméras et appareils photos des journalistes, qui se tiennent aux premiers rangs de l'assistance. Elle constitue un «premier acte » avant la scène centrale des graffitis sur les affiches.

Dans le cadre des «barbouillages », l'écriture exposée est une véritable ressource pour engager une confrontation avec le dispositif légal. L'inscription de slogans à la peinture sur des affiches publicitaires, acte illégal réalisé collectivement et en public permet une inculpation sur un motif précis du code pénal. La confrontation directe avec l'appareil répressif et judiciaire est finement insérée dans des principes d'actions non-violents et une mesure de chaque acte au regard de la loi. Les relais médiatiques permettent quant à eux une diffusion élargie de ces actions graphiques. Dans cette activité militante, la maitrise d'un dispositif graphique (tracts, graffitis, pancartes) est un moyen d'agir dans l'espace public en construisant concrètement un rapport de force par ajout (légal et illégal) d'inscriptions dans la ville. Le message inscrit sur les affiches reste volontairement basique. Il s'agit d'énoncer des principes clairs résumant une revendication complexe : la volonté affichée des militants anti-publicitaires n'est pas la disparition totale de la publicité, mais sa limitation. Il s'agit surtout de performer un effet graphique en faisant émerger l'idée selon laquelle il existe une pollution visuelle dans l'espace urbain, au même titre qu'une pollution atmosphérique ou sonore. Les slogans inscrits sur les affiches, orientés sur une critique de la société de consommation («Tais-toi et consomme», « La pub nous bouffe », « Consommer plus = penser moins ») ont progressivement évolué vers une exhortation à la prise de conscience environnementale ( ( Publicité $=$ pollution visuelle $»$ étant aujourd'hui le slogan de prédilection).

Cette performativité de l'écrit dans l'action anti-publicitaire repose toutefois sur une conception de l'écriture exposée comme totalement efficiente. Comme le souligne l'historienne Mireille Corbier au sujet de l'affichage à Rome, «l'écriture exposée à donc vocation à être lue. (...) Si une annonce a été faite en public (proscribere palam) en lettres bien distinctes (claris 
letteris), de façon à être lisible de plain-pied (unde de plano recte legi possit), non dans un lieu caché mais bien en évidence (...) en un lieu où un grand nombre de personnes peuvent le lire, on n'écoutera pas celui qui déclarera qu'il ne sait pas lire ou qu'il n'a pas regardé ce qui était affiché $»^{25}$. Or ce qui vaut pour l'affichage des lois dans la Rome antique s'appliquerait de la même façon pour l'affichage publicitaire des villes contemporaines : "Nuln'est censé ignorer ce qui est exposé dans l'espace public», semble affirmer l'historienne - que le message soit politique ou commercial. Mais elle souligne également que certaines inscriptions, haut perchées sur les frontons des monuments, n'ont pas pour vocation première d'être lisibles, mais seulement visibles - le citoyen sait que la loi est présente à cet endroit, dans toute sa matérialité, sans pour autant chercher à la déchiffrer. De la même manière, l'affichage publicitaire, malgré les stratégies de visibilité déployées par les afficheurs dans l'espace public, n'atteint pas forcément sa cible. Les militants anti-publicitaires développent ainsi une série de tactiques de non-lecture, en choisissant de porter leur regard sur tous les espaces où la publicité n'est pas présente (trottoirs, visages des passants, architecture, ciel...). Pourtant, ces tactiques reposent, une fois encore, sur l'attribution d'une force particulière à l'écrit et à l'image publicitaire - comme si voir une publicité et la lire revenait à intégrer, malgré soi, un message. Or cette efficacité de l'exposition, présentée comme une violence faite à l'esprit humain (le terme de « matraquage » employé par les militants en témoigne) reste relative. Le modèle du citadinlecteur qui s'imprégnerait telle une éponge des messages qu'on lui soumet - modèle à la fois construit par les publicitaires et les anti-publici- taires - n’est peut être pas aussi généralisé.

À travers l'observation des actions de «barbouillage », nous pouvons ainsi identifier comment cette force accordée à l'affichage publicitaire est à l'origine d'une contre-action d'écriture exposée tenant compte de savoirs sur les médias et la communication politique. Mais cette action ciblant spécifiquement l'affichage urbain ne prend pas en considération les phénomènes d'appropriation, de distance et de détournements à l'œuvre dans d'autres types de stratégies commerciales, s'appuyant sur les nouvelles technologies et la communication horizontale des consommateurs (le « buzz »). L'appel aux relais médiatiques et judiciaires des « barbouillages » est la preuve la plus éclairante que l'écriture exposée, seule, n'est pas assez instamment efficace. 
N

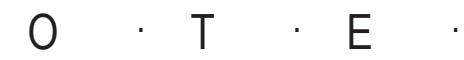

1. Dès les années 1960, suivant l'inspiration du mouvement situationniste, la contestation antipublicitaire fleurit sur les affiches. Le point d'orgue sera bien évidemment mai 1968, avec des slogans axés sur la problématique de l'aliénation des foules. En 1977, en Californie, le Billboard Liberation Front se fait également connaître par le détournement d'affiches publicitaires. Ce mouvement essaime en France (1984) avec les campagnes d'affichage de contre-publicité des Humains Associés, et au Canada (1989) avec Adbusters, qui promeut également des actions antipublicitaires.

2. Naomi Klein, No Logo: Taking Aim at the Brand Bullies, Toronto, Knopf Canada, 2000.

3. Laurent Jeanneau et Sébastien Lernould, Les nouveaux militants, Paris, Les Petits Matins, 2008.

4. Sébastien Darsy, Le Temps de l'Anti Pub, l'emprise de la publicité et ceux qui la combattent, Éditions Actes Sud, 2005.

5. Sur l'héritage de Mai 1968 et le rôle de l'écriture dans les modes d'action, voir Philippe Artières et Michèle Zancarini-Fournel (dir.), 68, une histoire collective (1962-1981), Paris, Éditions de La Découverte, 2008, notamment Philippe Artières, « “Je crie, j’écris". Quand la révolution passe par la prise de la parole et de l'écriture» et Béatrice Fraenkel « Les affiches en mai $68:$ l'atelier populaire des Beaux-Arts », dans ce même ouvrage.

6. Charles Tilly, La France conteste : de 1600 à nos jours, Fayard, Paris, 1986, p. 541.

7. Ibid, pp. 542-543.

8. Jürgen Habermas, L'espace public : archéologie de la publicité comme dimension constitutive de la société bourgeoise, Paris, Payot, 1978 [1962].

9. Isaac Joseph, Le passant considérable. Essai sur la dispersion de l'espace public, Paris, Librairie des Méridiens, 1984

10. Le travail présenté ici part d'une enquête ethnologique réalisée en 2002 sur les graffitis antipublicitaires dans le métro parisien et dans les rues de Paris. Cette enquête a été reprise en 2006 afin d'interroger l'évolution de cette activité militante qui consiste à inscrire à la bombe de peinture des slogans sur des affiches publicitaires, collectivement et publiquement.

11. John L. Austin, Quand dire, c'est faire, Paris, Seuil, 1970.

12. Béatrice Fraenkel, «Actes d'écriture: quand écrire, c'est faire. », Langage et Société, n 121-121, 2007, pp. 101-112

13. «Par écriture exposée, on entend n'importe quel type d'écriture conçu pour être utilisé dans des espaces ouverts, voire dans des espaces fermés, de façon à permettre la lecture à plusieurs (de groupe ou de masse) et à distance d'un texte écrit sur une surface exposée ; la condition nécessaire pour qu'il puisse être saisi est que l'écriture exposée soit de taille suffisante et qu'elle présente d'une manière suffisamment évidente et claire le message (des mots et / ou des images) dont elle est porteuse ", Armando Petrucci, Jeux de lettres, formes et usages de l'inscription en Italie 11è-20è siècle, Paris, Éditions de l'École des Hautes Études en Sciences Sociales, 1993.

14. L'inscription de slogans à la bombe de peinture sur une affiche publicitaire est considérée par le Code Pénal comme une « dégradation d'un bien appartenant à autrui » (articles 322-1 et 322-3).

15. Le terme de "déboulonneur», désignant une personne enlevant des boulons, est ici employé dans son sens métaphorique: «Ce collectif se propose de déboulonner la publicité, c'est-à-dire de la faire tomber de son piédestal, de détruire son prestige» 
(Manifeste des déboulonneurs, tract distribué lors des « barbouillages »).

16. Henry David Thoreau, De la désobéissance civile, Paris, Éditions des Mille et une nuits, 1996. Euvre posthume de 1866 dans laquelle l'auteur assume son incarcération pour refus de payer l'impôt à l'État américain, lui reprochant de soutenir l'esclavage et de mener une guerre contre le Mexique.

17. John Rawls, Théorie de la justice, Paris, Seuil, 1987, p. 405.

18. Erwing Goffman, Les cadres de l'expérience, Paris, Éditions de Minuit, 1991 [1974], p.19.

19. Ibid, p. 30.

20. Ibid, p. 52.

21. Ibid, p. 52.

22. Tract collecté le 28 octobre 2006.

23. L'article 322-1 du Code Pénal prévoit que « le fait de tracer des inscriptions, des signes ou des dessins, sans autorisation préalable, sur les façades, les véhicules, les voies publiques ou le mobilier urbain est puni de 3750 euros d'amende et d'une peine de travail d'intérêt général lorsqu'il n'en est résulté qu'un dommage léger ».

24. Loi n²004-204 du 9 mars 2004.

25. Mireille Corbier, Donner à voir, donner à lire. Mémoire et communication dans la Rome ancienne, Paris, Éditions du CNRS, 2006, p. 47.

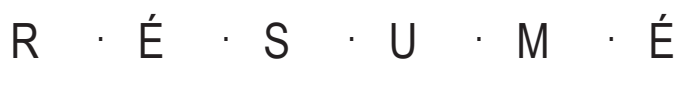

Cet article propose une analyse du phénomène des graffitis anti-publicitaires à travers une enquête réalisée auprès du collectif des Déboulonneurs, rassemblant des militants spécialisés dans les actions médiatiques réalisées à visage découvert. L'étude d'une action spécifique mise en œuvre par ce collectif (les « barbouillages ») met en évidence la façon dont ces militants inscrivent leur lutte contre ce qui est désigné comme une «pollution visuelle » dans l'espace physique de la ville. Les « barbouillages », dont le but officiel est de modifier la loi sur l'affichage publicitaire, sont construits comme des événements publics. Deux aspects de cette mise en scène de l'écrit dans l'espace urbain seront ici explorés. D'une part, dans le rapport aux forces de l'ordre, dont la présence systématique est prise en considération à travers l'énonciation de principes de non-violence. D'autre part, dans la circulation de l'écriture exposée contestataire, maîtrisée à destination des médias. Nous verrons enfin comment ces actions reposent sur l'attribution d'une force à l'écriture exposée.

\begin{abstract}
This article proposes an analysis of the phenomenon of anti-advertising graffitis, through an inquiry carried out within the collective of Déboulonneurs. This group assembles french activists specialized in openly realized media actions. The study of a specific action by this collective (the "scribblings") highlights the way these activists expose their fight against a "visual pollution" in the physical space of cities. Those "scribblings", which official goal is to modify advertising laws, are constructed as public events. Two aspects of this writing performance in the urban space will be investigated here. On one hand, the relationship
\end{abstract}


with police forces, whose systematic presence is taken into consideration by setting nonviolence principles. On the other hand, the circulation of protest writings, especially designed for the media. Finally, we will see how these actions are based on the attribution of a strength to the exposed writing. 